\title{
A Branch Current Reallocation Based Energy Balancing Strategy for the Modular Multilevel Matrix Converter Operating Around Equal Frequency
}

\author{
Boran Fan ${ }^{1}$, Kui Wang ${ }^{1}$, Chunyang Gu ${ }^{2}$, Pat Wheeler ${ }^{2}$, Yongdong $\mathrm{Li}^{1}$ \\ ${ }^{1}$ State Key Lab of Power System, Dept. of Electrical Engineering, Tsinghua University, Beijing, China \\ ${ }^{2}$ Department of Electrical and Electronic Engineering, University of Nottingham, Nottingham, United Kingdom \\ E-mail: fbr13@mails.tsinghua.edu.cn
}

\begin{abstract}
Modular multilevel matrix converter (M3C) is a promising topology for medium-voltage high-power applications. Due to the modular structure, it features easy scalability, high quality output waveforms and superior fault tolerance. However, M3C suffers serious capacitor-voltage fluctuation if the output frequency gets closer to the input frequency. This limits its use in the adjustable-speed-drive (ASD) applications. This paper introduces a theoretical analysis in phasor-domain to find the branch energy equilibrium point of $\mathrm{M3C}$ when operating around equal frequency. On the basis of this equilibrium point, a branch current reallocation based energy balancing control method is proposed to equalize the energy stored in the nine converter branches. With this novel control method, $\mathrm{M}^{3} \mathrm{C}$ can effectively overcome the capacitor voltage fluctuation with neither using common voltage nor applying reactive power at the input side.
\end{abstract}

Keywords-modular multilevel matrix converter (M3C); energy and balancing control; equal frequency

\section{INTRODUCTION}

The modular multilevel matrix converter (M3C), shown in Fig. 1, connects two three-phase systems (input-side and output-side) by nine branches. Each branch is a cascaded connection of full-bridge (H-bridge) converter cells. It enables direct $\mathrm{AC}-\mathrm{AC}$ bidirectional power conversion and ensures three-phase input and output currents sinusoidal with any power factor [1]-[4]. Like other members of the modular multilevel cascade converter (MMCC) family, the voltage ratings of $\mathrm{M} 3 \mathrm{C}$ can be enhanced easily by increasing the number of cascaded cells. M3C is more suitable for lowspeed constant-torque motor drives compared with the modular multilevel converters (MMC) in back-to-back configuration [5], [6]. These advantages make M3C a promising topology for the medium-voltage high-power adjustable-speed-drive (ASD) application. However, M3C suffers serious capacitor-voltage fluctuation if the output frequency gets closer to the input frequency. This is caused by a very low-frequency (the frequencies' difference between two three-phase system) reactive power on the branch. This reactive power becomes constant when both systems are with identical frequency. It causes power difference among branches and as a result, unbalances the energy stored in different branches.

In order to solve this problem, [2] presents a solution by both injecting circulating current into $\mathrm{M} 3 \mathrm{C}$ and applying reactive power at the input side. However, a reactive power at the input side is not allowed in some cases as input side is mostly connected to the grid. Reference [4] introduces common voltage to avoid reactive power at the input side which is similar to the mitigation control of the MMC at low-speed range [7]. However, the reference common voltage is difficult to design and may cause over-modulation. The common voltage may also lead to premature failure of motor bearings. Reference [8] adjusts the motor voltage to ensure the input and output side share a same voltage magnitude. This helps to achieve lower branch currents. But this method has a few restrictions and also needs to apply reactive power at the input side.

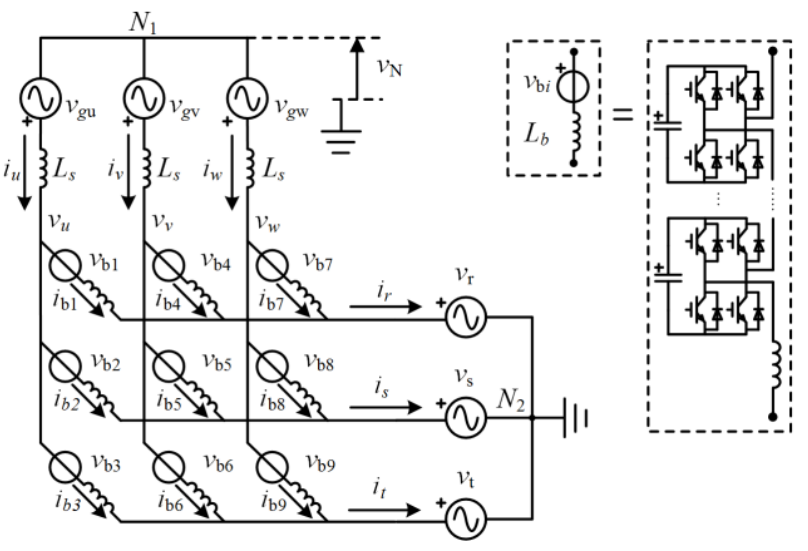

Fig. 1. Circuit Configuration of the M3C

As the reactive power at the input side is not allowed in some conditions and the common voltage may cause serious problems, this paper attempts to develop a control method only using circulating currents in the M3C to equalize the energy of the nine branches. This paper firstly proves the availability of this consideration. Then it develops a strategy to design specifically appropriate circulating currents. With this control method, M3C can effectively overcome the capacitor voltage fluctuation with neither using common voltage nor applying reactive power at the input side. The proposed control strategy has been validated by simulation and experiment.

\section{BASIC THEORY OF THE M3C}

\section{A. Double $\alpha \beta 0$ Transformation}

In the $\mathrm{M} 3 \mathrm{C}$, there are four independent inner circulating currents [1]-[4]. These circulating currents are independent of input side currents $\left(i_{\mathrm{u}}, i_{\mathrm{v}}, i_{\mathrm{w}}\right)$ and output side currents $\left(i_{\mathrm{r}}, i_{\mathrm{s}}\right.$, $i_{\mathrm{t}}$ ). Power transfer between different branches is realized by adjusting the value of these 4 circulating currents. Theoretically, there are multiple definitions of these circulating currents. In recent literatures, by introducing a so-called double $\alpha \beta 0$ transformation to $\mathrm{M} 3 \mathrm{C}$, circulating 
currents are defined as $i_{\alpha \alpha}, i_{\alpha \beta}, i_{\beta \alpha}$ and $i_{\beta \beta}$. The definition of the double $\alpha \beta 0$ transformation $\mathrm{T}_{\text {Dual- } \alpha \beta}$ is in (1).

$$
\mathbf{T}_{\text {Dual- } \alpha \beta}\left(\mathbf{M}_{3 * 3}\right)=\mathbf{T}_{\mathrm{ab}} \cdot \mathbf{M}_{3 * 3} \cdot \mathbf{T}_{\mathrm{ab}} \mathbf{T}^{\mathbf{T}}, \quad \mathbf{T}_{\mathrm{ab}}=\left[\begin{array}{ccc}
\frac{2}{3} & -\frac{1}{3} & -\frac{1}{3} \\
0 & \frac{\sqrt{3}}{3} & -\frac{\sqrt{3}}{3} \\
\frac{1}{3} & \frac{1}{3} & \frac{1}{3}
\end{array}\right]
$$

Perform the double $\alpha \beta 0$ transformation on nine branch currents and gets the $i_{\alpha \alpha}, i_{\alpha \beta}, i_{\beta \alpha}$ and $i_{\beta \beta}$ in (2). Here $i_{\alpha 0}$ and $i_{\beta 0}$ are components of the input currents on the $\alpha$-axis and $\beta$-axis and $i_{\alpha 0}$ and $i_{\beta 0}$ are components of the output currents on the $\alpha$-axis and $\beta$-axis.

$$
\left[\begin{array}{lll}
i_{\alpha \alpha} & i_{\alpha \beta} & i_{\alpha 0} \\
i_{\beta \alpha} & i_{\beta \beta} & i_{\beta 0} \\
i_{0 \alpha} & i_{0 \beta} & i_{00}
\end{array}\right]=\mathbf{T}_{\mathbf{a b}}\left[\begin{array}{lll}
i_{b 1} & i_{b 2} & i_{b 3} \\
i_{b 4} & i_{b 5} & i_{b 6} \\
i_{b 7} & i_{b 8} & i_{b 9}
\end{array}\right] \mathbf{T}_{\mathbf{a b}}^{\mathbf{T}}
$$

The double $\alpha \beta 0$ transformation also helps to realize a decoupled control of these four circulating currents as shown in Fig. 2 [9]. Using simple proportional-integral (PI) regulators, the $i_{\alpha \alpha}, i_{\alpha \beta}, i_{\beta \alpha}$ and $i_{\beta \beta}$ are easily controlled by $v_{\alpha \alpha}$, $v_{\alpha \beta}, v_{\beta \alpha}$ and $v_{\beta \beta}$. Here, voltage $v_{\alpha \alpha}, v_{\alpha \beta}, v_{\beta \alpha}$ and $v_{\beta \beta}$ are defined in (3).

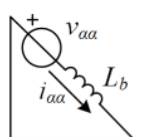

(a)

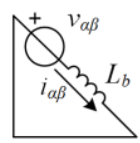

(c)

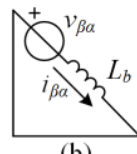

(b)

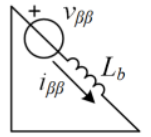

(d)

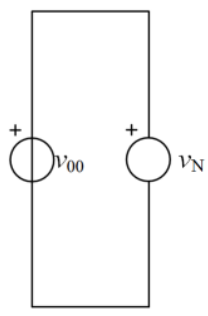

(e)
Fig. 2. Circulating currents and common-voltage in the M3C

$$
\left[\begin{array}{ccc}
v_{\alpha \alpha} & v_{\alpha \beta} & v_{\alpha 0} \\
v_{\beta \alpha} & v_{\beta \beta} & v_{\beta 0} \\
v_{0 \alpha} & v_{0 \beta} & v_{00}
\end{array}\right]=\mathbf{T}_{\mathbf{a b}}\left[\begin{array}{ccc}
v_{b 1} & v_{b 2} & v_{b 3} \\
v_{b 4} & v_{b 5} & v_{b 6} \\
v_{b 7} & v_{b 8} & v_{b 9}
\end{array}\right] \mathbf{T}_{\mathbf{a b}}^{\mathbf{T}}
$$

The value of the common voltage $v_{\mathrm{N}}$ in Fig. 1 is equal to the value of $v_{00}$ as shown in Fig. 2. In order to avoid common-voltage, according to (1) and (3), the value of $v_{00}$ satisfies (4).

$$
v_{N}=v_{00}=\frac{1}{9} \sum_{i=1}^{9} v_{b i} @ 0
$$

\section{B. Basic Branch Current Allocation}

If control circulating currents $i_{\alpha \alpha}, i_{\alpha \beta}, i_{\beta \alpha}$ and $i_{\beta \beta}$ all to be zero, the nine branch currents would be (5). The branch current consists of $1 / 3$ of the $x$-phase $(x=\mathrm{u}, \mathrm{v}, \mathrm{w})$ input side current and $1 / 3$ of the $y$-phase $(y=\mathrm{r}, \mathrm{s}, \mathrm{t})$ output side current.

$$
\left[\begin{array}{lll}
i_{b 0,1} & i_{b 0,2} & i_{b 0,3} \\
i_{b 0,4} & i_{b 0,5} & i_{b 0,6} \\
i_{b 0,7} & i_{b 0,8} & i_{b 0,9}
\end{array}\right]=\frac{1}{3} \cdot\left[\begin{array}{ccc}
i_{u} & i_{u} & i_{u} \\
i_{v} & i_{v} & i_{v} \\
i_{w} & i_{w} & i_{w}
\end{array}\right]+\frac{1}{3} \cdot\left[\begin{array}{ccc}
i_{r} & i_{s} & i_{t} \\
i_{r} & i_{s} & i_{t} \\
i_{r} & i_{s} & i_{t}
\end{array}\right]
$$

In this paper, the branch currents in (5) are defined as the "basic branch current". Recent literatures mostly assume this branch current allocation as the branch energy equilibrium point. On the basis of this allocation, circulating currents $i_{\alpha \alpha}, i_{\alpha \beta}, i_{\beta \alpha}$ and $i_{\beta \beta}$ are designed to compensate possible branch energy difference.

\section{Capacitor-voltage Fluctuation}

In this paper, the input and output systems are assumed to be three-phase balanced and with positive sequence. No reactive power is applied at the input side. Voltages and currents at the input and output side are defined as,

$$
\begin{aligned}
& v_{u}=\hat{v}_{m 1} \cos \left(\omega_{1} t\right) \\
& i_{u}=\hat{i}_{m 1} \cos \left(\omega_{1} t\right) \\
& v_{r}=\hat{v}_{m 2} \cos \left(\omega_{2} t+\theta\right) \\
& i_{r}=\hat{i}_{m 2} \cos \left(\omega_{2} t+\theta-\varphi\right)
\end{aligned}
$$

Applying the basic branch currents allocation defined in (5), the power on branch 1, for instance, is shown in (10). This branch power consists of frequency components of $\omega_{1^{-}}$ $\omega_{2}, \omega_{1}+\omega_{2}, 2 \omega_{1}, 2 \omega_{2}$

$$
\begin{aligned}
& p_{b 1}=\left(v_{u}-v_{r}\right) \cdot\left(i_{u}+i_{r}\right) / 3
\end{aligned}
$$

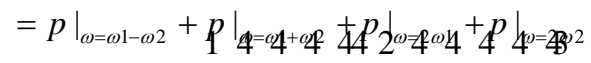

$$
\begin{aligned}
& p_{h}
\end{aligned}
$$

When output side frequency $\left(\omega_{2}\right)$ gets close to the input side frequency $\left(\omega_{1}\right)$, the low-frequency power component of $\omega_{1}-\omega_{2}$ causes large branch energy fluctuation. This power component is shown in (11) and (12). In conclusion, when operating $\mathrm{M} 3 \mathrm{C}$ around equal frequency, applying the basic branch currents allocation causes large branch energy fluctuation. Therefore a branch currents reallocation should be developed.

$$
\begin{aligned}
& \left.p\right|_{\omega=\omega 1-\omega 2}= \\
& \frac{\sqrt{\hat{v}_{m 1}^{2} \hat{i}_{m 2}^{2}+\hat{v}_{m 2}^{2} \hat{i}_{m 1}^{2}-2 \hat{v}_{m 1} \hat{v}_{m 2} \hat{i}_{m 1} \hat{i}_{m 2} \cos \varphi}}{6} \cdot \cos \left[\left(\omega_{1}-\omega_{2}\right) t-\theta+\delta\right]
\end{aligned}
$$

$$
\delta=\arctan \left(\frac{\hat{v}_{m 1} \hat{i}_{m 2} \sin \varphi}{\hat{v}_{m 1} \hat{i}_{m 2} \cos \varphi-\hat{v}_{m 2} \hat{i}_{m 1}}\right)
$$

\section{BRANCH ENERGY EQUILIBRIUM POINT OF M3C WHEN OPERATING AROUND EQUAL FREQUENCY}

\section{A. Phasor-domain Analysis of the $M 3 C$}

In this paper, analysis is performed in phasor-domain instead of time-domain. This helps to visualize the analysis of branch energy balancing for the equal-frequency operation. Assuming $\omega_{1}=\omega_{2}$, rewritten the definition of (6)(9) in phasor-domain,

$$
\begin{aligned}
& {\stackrel{\mathrm{r}}{v_{u}}}=\hat{v}_{m 1} \cdot e^{j \cdot 0} \\
& \stackrel{\mathrm{r}}{i}_{u}=\hat{i}_{m 1} \cdot e^{j \cdot 0} \\
& \stackrel{\mathrm{r}}{v_{r}}=\hat{v}_{m 2} \cdot e^{j \cdot \theta} \\
& \stackrel{\mathrm{r}}{i_{r}}=\hat{i}_{m 2} \cdot e^{j \cdot(\theta-\varphi)}
\end{aligned}
$$

The basic branch currents in phasor-domain would be, 


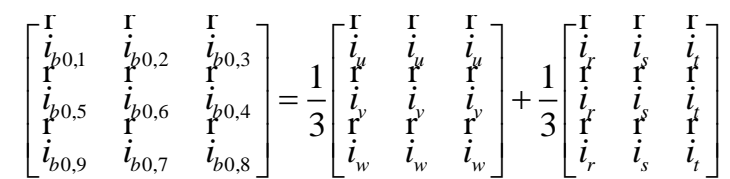

In (18), $\stackrel{\mathrm{I}}{e}_{n i}(i=1,2, . .9)$ is the unit length phasor that leads branch voltage $\stackrel{\mathrm{I}}{b i}_{b i}(i=1,2, . .9)$ by $90^{\circ}$ as in (19).

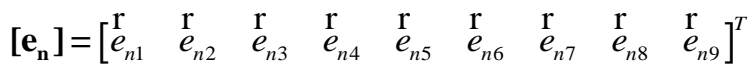

$$
\begin{aligned}
& \stackrel{\mathrm{r}}{e_{n i}}=\frac{\stackrel{\mathrm{r}}{v_{b i}}}{\left|\mathrm{r}_{b i}\right|} \cdot j @ e^{j \cdot \sigma_{b i}}=\cos \sigma_{b i}+j \cdot \sin \sigma_{b i},(i=1,2, . .9)
\end{aligned}
$$

Here $\left[\mathbf{e}_{\mathbf{n}}\right]$ is separated into three groups as $\left[\mathbf{e}_{\mathbf{n} 1,5,9}\right]$, $\left[\mathbf{e}_{\mathbf{n} 2,6,7}\right]$ and $\left[\mathbf{e}_{\mathbf{n} 3,4,8}\right]$. In each group, $\stackrel{\mathbf{I}}{e}_{n 1} / \stackrel{\mathbf{I}}{e}_{n 2} / \stackrel{\mathbf{I}}{e}_{n 3}$ leads

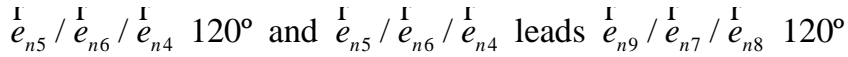
respectively as in (20)-(22).

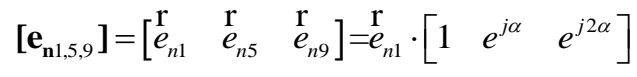

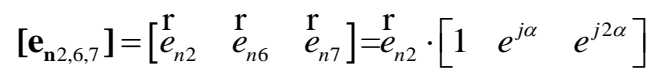

$$
\begin{aligned}
& {\left[\mathbf{e}_{\mathbf{n} 3,4,8}\right]=\left[\begin{array}{lll}
\stackrel{r}{e}_{n 3} & \stackrel{\mathrm{r}}{e}_{n 4} & \stackrel{\mathrm{r}}{e}_{n 8}
\end{array}\right]={\stackrel{\mathrm{r}}{e_{n 3}}}_{n} \cdot\left[\begin{array}{lll}
1 & e^{j \alpha} & e^{j 2 \alpha}
\end{array}\right]}
\end{aligned}
$$

Fig. 3 is the phasor diagram of the $\mathrm{M} 3 \mathrm{C}$, input side voltage and currents in (13) and (14) are shown in red color; output side voltage and currents in (15) and (16) are shown in green color; basic branch currents in (17) are shown in purple color; $\left[\mathbf{e}_{\mathbf{n}}\right]$ are shown in blue color. Obviously, in order to stabilize branch energies, there should be no active power on each branch. Therefore the branch current phasor $i_{b i}(i=1,2, . .9)$ should be in the same or opposite direction with $\stackrel{\mathrm{I}}{e}_{n i}(i=1,2, . .9)$. This condition is explain in (23)-(25). Here $[\mathbf{C}]$ is the vector of nine branch currents magnitude.

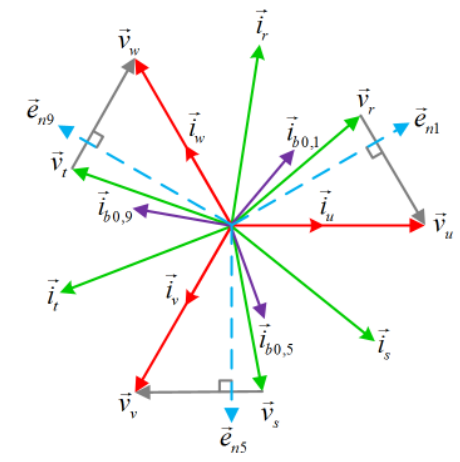

(a)

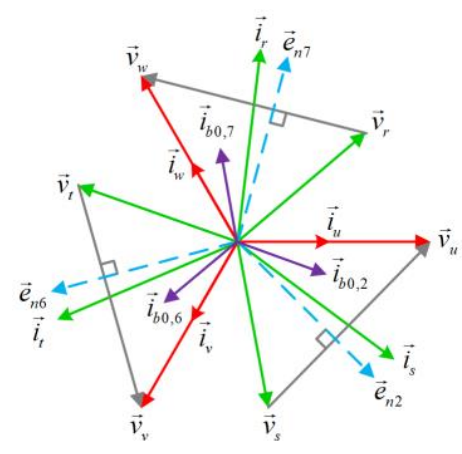

(b)

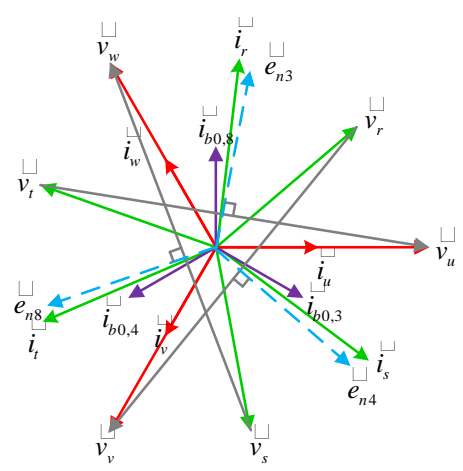

(c)

Fig. 3. Phasor Diagram of the M3C

(a) Branch 1,5,9 (b) Branch 2,6,7 (c) Branch 3,4,8

However, in Fig. 3 the basic branch current phasors are clearly with phase difference to $\left[\mathbf{e}_{\mathbf{n}}\right]$ and do not meet the condition in (23). This causes a branch energy derivation when $\omega_{1}=\omega_{2}$ and a large branch energy fluctuation when $\omega_{1} \approx \omega_{2}$. This result is consist with the analysis in timedomain as explain in PART II.C.

$$
\left[\mathbf{i}_{\mathbf{b}}\right]=[C] \cdot\left[\mathbf{e}_{\mathbf{n}}\right]
$$

$$
\begin{aligned}
& {\left[\mathbf{i}_{\mathbf{b}}\right]=\left[\begin{array}{lllllllll}
\stackrel{r}{i}_{b 1} & \stackrel{r}{i}_{b 2} & \stackrel{r}{i}_{b 3} & \stackrel{r}{i}_{b 4} & \stackrel{r}{i}_{b 5} & \stackrel{r}{i}_{b 6} & \stackrel{r}{i}_{b 7} & \stackrel{r}{i}_{b 8} & \stackrel{r}{i}_{b 9}
\end{array}\right]^{T}} \\
& {[\mathbf{C}]=\left[\begin{array}{lllllllll}
c_{1} & c_{2} & c_{3} & c_{4} & c_{5} & c_{6} & c_{7} & c_{8} & c_{9}
\end{array}\right]}
\end{aligned}
$$

\section{B. Branch Current Magnitude Calculation}

As input and output side are three-phase balanced, the value of branch current magnitudes $c_{1}, c_{5}$ and $c_{9}$ are equal and so as $c_{2}, c_{6}, c_{7}$ and $c_{3}, c_{4}, c_{8}$ as shown in (26).

$$
c_{1}=c_{5}=c_{9}, c_{2}=c_{6}=c_{7}, c_{3}=c_{4}=c_{8}
$$

Branch currents satisfy,

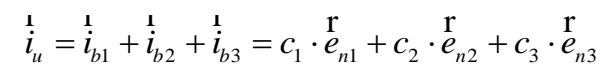

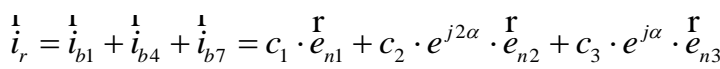

Combined with (14) and (16), the branch currents magnitudes satisfy,

$$
[\mathrm{A}][\mathrm{C}]=[\mathrm{B}]
$$

Where,

$$
\begin{aligned}
& {[\mathbf{A}]=\left[\begin{array}{ccc}
\sin \sigma_{b 1} & \sin \sigma_{b 2} & \sin \sigma_{b 3} \\
\sin \sigma_{b 1} & \sin \left(\sigma_{b 2}+2 \alpha_{n}\right) & \sin \left(\sigma_{b 3}+\alpha_{n}\right) \\
\cos \sigma_{b 1} & \cos \left(\sigma_{b 2}+2 \alpha_{n}\right) & \cos \left(\sigma_{b 3}+\alpha_{n}\right)
\end{array}\right]} \\
& {[\mathbf{B}]=\left[\begin{array}{lll}
0 & \hat{i}_{m 2} \cdot \sin (\theta-\varphi) & \hat{i}_{m 2} \cdot \cos (\theta-\varphi)
\end{array}\right]}
\end{aligned}
$$

Branch current magnitudes are obtained by solving this equation. Assuming $\left[\mathbf{C}^{*}\right.$ is a possible solution of (29), combined with (23) the reallocated branch currents should be $\left[\mathbf{i}_{\mathbf{b}}\right]^{*}=[\mathbf{C}]^{*} \cdot\left[\mathbf{e}_{\mathbf{n}}\right]$. In this paper, $\left[\mathbf{i}_{\mathbf{b}}\right]=\left[\mathbf{i}_{\mathbf{b}}\right]^{*}$ is called as the branch energy equilibrium point of $\mathrm{M} 3 \mathrm{C}$ when operating around equal frequency 


\section{Disscussion on the Control Availability}

This paper attempts to develop a control method only using circulating currents in the $\mathrm{M} 3 \mathrm{C}$ to equalize the energy of the nine branches. The availability of this consideration is equivalent to the solvability of the linear equation in (29).

Assume modulation rate $\mathrm{m}$ as,

$$
m=\hat{v}_{m 2} / \hat{v}_{m 1} \geq 0
$$

It is proved that the mentioned consideration is possible under following conditions:

$\checkmark \quad m \neq 1$

In this condition, the matrix [A] is invertible. The reallocated circulating currents would be

$$
\left[\mathbf{i}_{\mathbf{b}}\right]^{*}=[\mathbf{A}]^{-1} \cdot[\mathbf{B}] \cdot\left[\mathbf{e}_{\mathbf{n}}\right]
$$

$$
\checkmark \quad m=1, \sin \varphi=0
$$

In this condition, (29) develops multiple solutions. The basic current allocation is one of the solution. Take branches 1, 5 and 9 for instance, as shown in Fig. 4, condition (23) holds because branch currents $\stackrel{1}{i}_{b 1}, \stackrel{1}{i}_{b 5}$ and $\stackrel{1}{i}_{b 9}$ are in the same or opposite direction with $\stackrel{\stackrel{I}{e}}{n 1},_{e_{n 5}}^{\stackrel{I}{e}}$ and $\stackrel{\mathfrak{I}}{e}_{n 9}$ respectively.

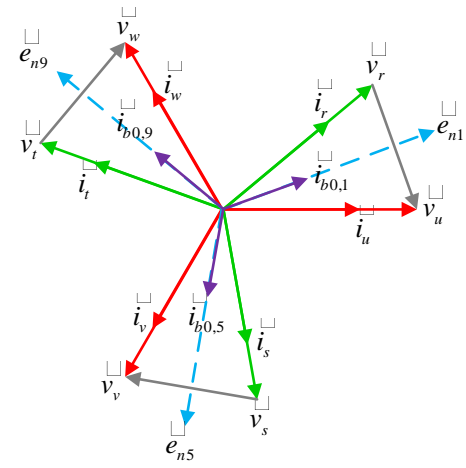

Fig. 4. Phasor Diagram of $m=1, \sin \varphi=0$

$$
\checkmark \quad m=1, \sin \varphi \neq 0, \theta=0 \text { or }-120^{\circ} \text { or } 120^{\circ}
$$

This condition consists of three special cases. Take $\theta=0$ for an instance. In this case, if short branches 1, 5 and 9 and block other branches, there would not be any branch energy fluctuation. For cases of $\theta=-120^{\circ}$ and $\theta=120^{\circ}$, the shorted branches should be $2,6,7$ and $3,4,8$ respectively.

\section{Impossible condition:}

- $\quad m=1, \sin \varphi \neq 0, \theta \neq 0,-120^{\circ}, 120^{\circ}$

The mentioned consideration is impossible under this condition. In this case, reactive power or the commonvoltage are necessary for the mitigation of the capacitorvoltage fluctuation.

\section{PROPOSED CONTROL METHOD}

According to PART III.C, if the voltage magnitude of the input side and output side are not identical, the application of branch reallocation in (33) overcomes the capacitor-voltage fluctuation when output frequency gets close to the input frequency.

In this paper, the theoretical calculation process in phasor-domain explained in PART III is redesigned into a real-time control method. The control block is shown in Fig. 5 . Here an added branch power compensation control is complemented as shown in dashed frame in Fig. 5. The directions of $\left[\mathbf{e}_{\mathbf{n}}\right]$ are slightly adjusted to regulate branch energies. Compared to existing control method, the proposed control do not design circulating currents on the basis of basic branch currents. Instead, it firstly moves the M3C close to the branch energy equilibrium point by applying the reallocated branch currents. Then the control method slightly adjusts circulating currents to realize a final branch energy equalization.

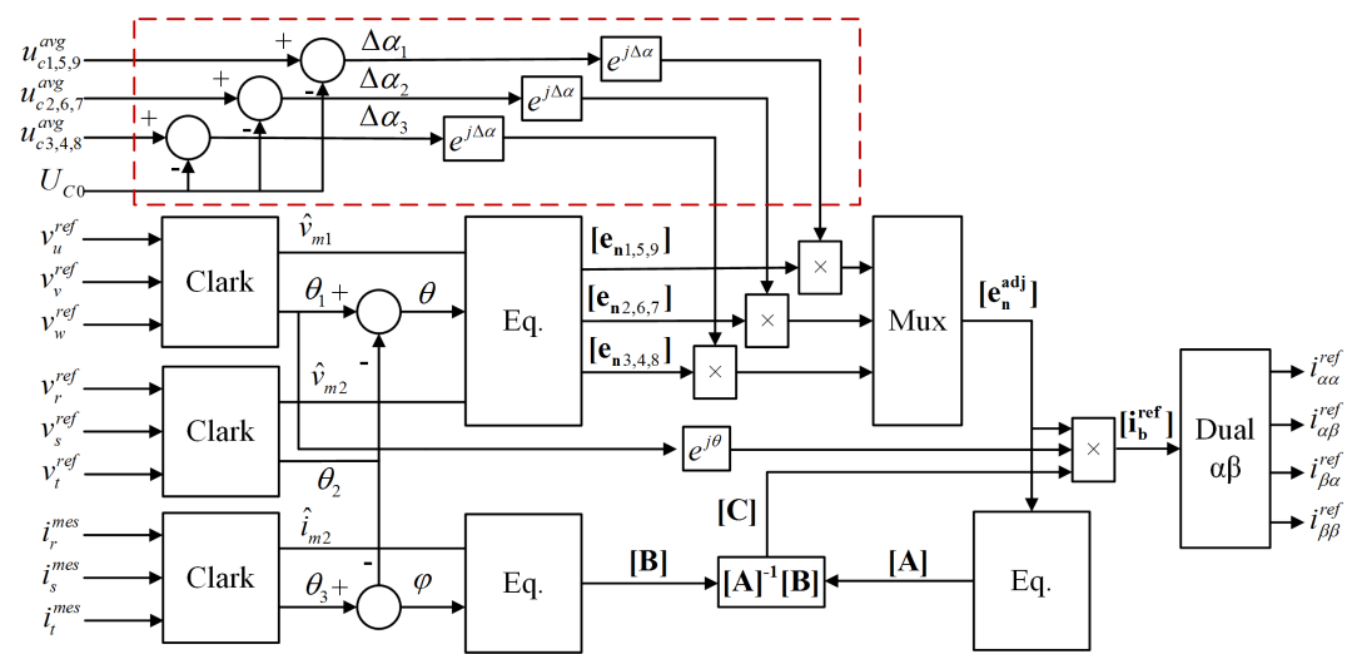

Fig. 5. Proposed Control for the M3C

\section{Simulation AND EXPERIMENT RESUltS}

\section{A. Simulation results}

To verify the theoretical analysis and the proposed control method, simulations studies are conducted in PLECS environment. Simulation parameters are shown in Tab. 1. In Fig. 1, the input side of the $\mathrm{M} 3 \mathrm{C}$ is connected to the grid and the output side is connected to a RL-load.

TABLE I. SIMULATION PARAMETERS

Parameters $\quad$ Symbols $\quad$ Value




\begin{tabular}{lll}
\hline Switching Frequency & $f_{\mathrm{s}}$ & $2 \mathrm{kHz}$ \\
Sub-modules per branch & $\mathrm{N}$ & 1 \\
Module Capacitance & $\mathrm{C}$ & $1 \mathrm{mF}$ \\
Branch inductance & $L_{\mathrm{b}}$ & $5 \mathrm{mH}$ \\
Grid-connected inductance & $L_{\mathrm{s}}$ & $5 \mathrm{mH}$ \\
Capacitor Voltage & $\mathrm{U}_{\mathrm{C} 0}$ & $500 \mathrm{~V}$ \\
Input frequency & $f_{1}$ & $50 \mathrm{~Hz}$ \\
Input Voltage Magnitude & $\hat{v}_{m 1}$ & $220 \mathrm{~V}$ \\
Output Voltage Magnitude & $\hat{v}_{m 2}$ & $150 \mathrm{~V}$ \\
Output Current Magnitude & $\hat{i}_{m 2}$ & $20 \mathrm{~A}$ \\
\hline \hline
\end{tabular}

The validity of the proposed control strategy is verified under different operating conditions. Six test operating conditions are set up in Tab .2. The output frequency $f_{2}$ is set to be identical or near to the input frequency. Reactive power is applied at the output side.

TABLE II. TEST CONDITIONS OF THE PROPOSED CONTROL

\begin{tabular}{ccc}
\hline \hline Test Condition & $\boldsymbol{f}_{\mathbf{2}}$ & $\cos \varphi$ \\
\hline $\mathbf{a}$ & $50 \mathrm{~Hz}$ & 1 \\
$\mathbf{b}$ & $50 \mathrm{~Hz}$ & 0.5 \\
$\mathbf{c}$ & $49 \mathrm{~Hz}$ & 1 \\
$\mathbf{d}$ & $49 \mathrm{~Hz}$ & 0.5 \\
$\mathbf{e}$ & $51 \mathrm{~Hz}$ & 1 \\
$\mathbf{f}$ & $51 \mathrm{~Hz}$ & 0.5 \\
\hline \hline
\end{tabular}

Corresponding simulation results are presented in Fig. 6. In Fig. 6 before $0.4 \mathrm{~s}$, the basics current allocation is applied. At $0.4 \mathrm{~s}$, the proposed control strategy is added and the capacitor voltage fluctuation is effectively suppressed in \pm $15 \mathrm{~V}$ in around $0.1 \mathrm{~s}$.

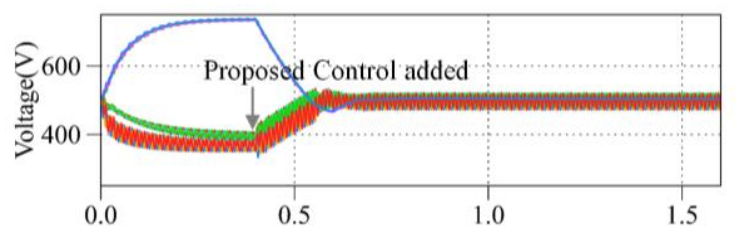

(a) Time (s)

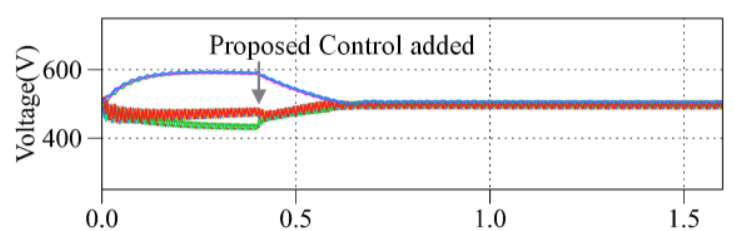

(b) Time (s)

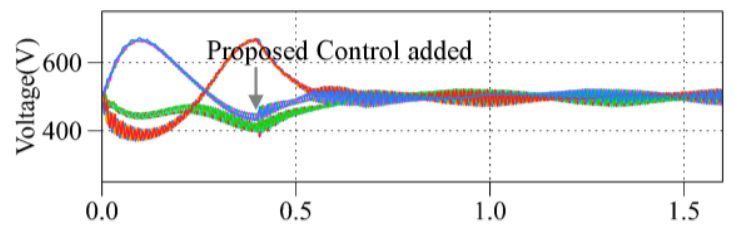

(c) Time (s)

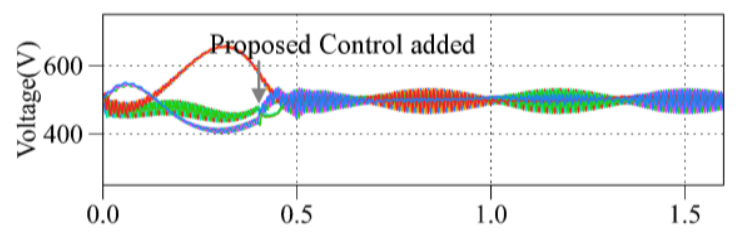

(d) Time (s)

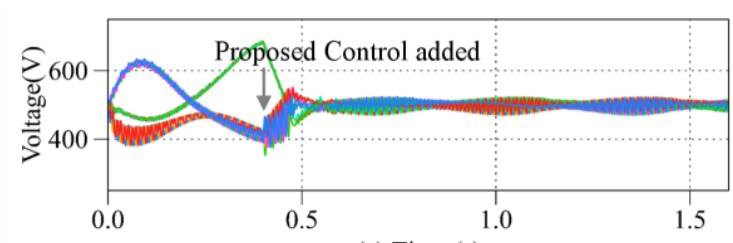

(e) Time (s)

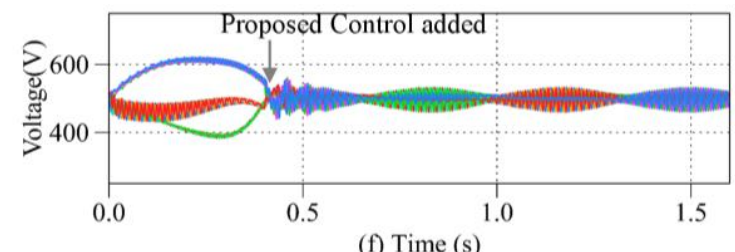

Fig. 6 Nine Branch Capacitor Voltages

(a) $f_{2}=50 \mathrm{~Hz}, \cos \varphi=1$, (a) $f_{2}=50 \mathrm{~Hz}, \cos \varphi=0.5$, (a) $f_{2}=49 \mathrm{~Hz}$, $\cos \varphi=1$, (a) $f_{2}=49 \mathrm{~Hz}, \cos \varphi=0.5$, (a) $f_{2}=51 \mathrm{~Hz}, \cos \varphi=1$, (a) $f_{2}=$ $51 \mathrm{~Hz}, \cos \varphi=0.5$

The proposed control do not apply reactive power at the input side. For instance, under test condition (b), input voltages (grid voltages), input currents and branch currents are shown in Fig. 7. As the input voltages are in-phase with the output currents, it proves no reactive power at the input side.
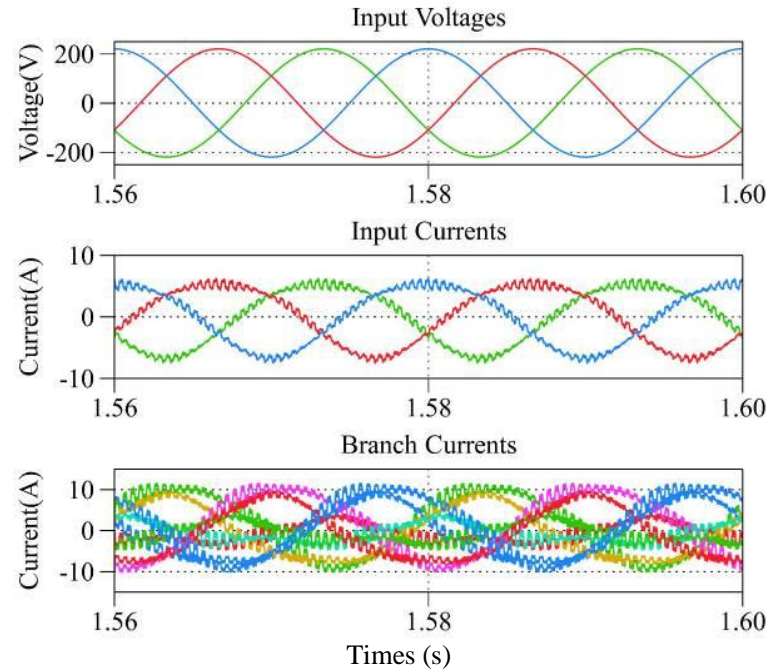

Fig. 7. Simulation results under test condition (b)

\section{B. Experiment results}

A $\mathrm{M} 3 \mathrm{C}$ experiment platform, shown in Fig. 8 is established with the same parameters in Tab. 1. Experiment results will be presented in the final paper. 


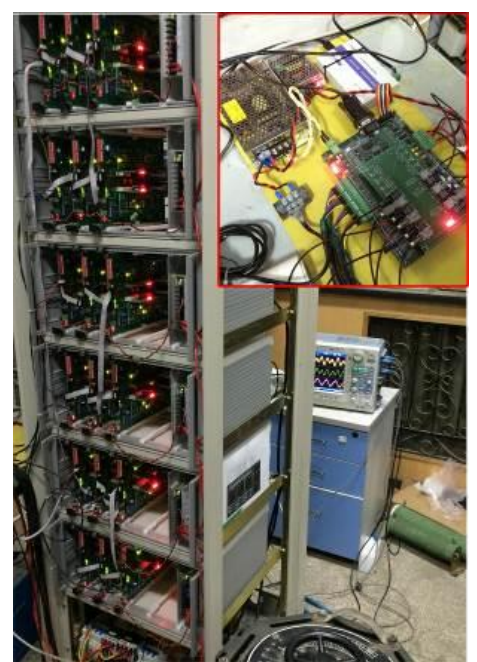

Fig. 8. The M3C Experiment Platform

\section{CONCLUSION}

This paper performs a theoretical analysis in phasordomain to find the branch energy equilibrium point of the $\mathrm{M} 3 \mathrm{C}$ when operating around equal frequency. On the basis of this equilibrium point, a branch current reallocation based energy balancing control strategy is proposed. The availability of the control strategy is proved. With this control strategy, M3C can effectively overcome the capacitor voltage fluctuation with neither using common voltage nor applying reactive power at the input side.

\section{REFERENCES}

[1] F. Kammerer, J. Kolb, and M. Braun, "Fully decoupled current control and energy balancing of the modular multilevel matrix converter," in Conf. Rec. IEEE-EPE-PEMC 2012, LS2a.3.

[2] W. Kawamura, M. Hagiwara, and H. Akagi, "A broad range of frequency control for the modular multilevel cascade converter based on triple-star bridge-cells (MMCC-TSBC)," in Conf. Rec. IEEEECCE 2013, pp. 4014-4021.

[3] W. Kawamura, M. Hagiwara, and H. Akagi, "Control and experiment of a 380-V, 15-kW motor drive using modular multilevel cascade converter based on triple-star bridge cells (MMCC-TSBC)," in Proc. IPEC 2014, pp. 3742-3749

[4] F. Kammerer, M. Gommeringer, J. Kolb, and M. Braun, "Energy balancing of the modular multilevel matrix converter based on a new transformed arm power analysis," in Conf. Rec. EPE 2014, pp. 1-10.

[5] K. Ilves, L. Bessegato, and S. Norrga, "Comparison of cascaded multilevel converter topologies for ac/ac conversion," in Conf. Rec. IPEC 2014, pp. 1087-1094.

[6] Y. Okazaki, W. Kawamura, M. Hagiwara, H. Akagi, T. Ishida, M. Tsukakoshi, and R. Nakamura, "Which is more suitable for MMCCbased medium-voltage motor drives, a DSCC inverter or a TSBC converter?," in Conf. Rec. ICPE 2015, WeA2-1.

[7] K. Wang, Y. Li, Z. Zheng, and L. Xu, "Voltage Balancing and Fluctuation-Suppression Methods of Floating Capacitors in a New Modular Multilevel Converter," IEEE Transactions on Industrial Electronics, vol. 60, no. 5. pp. 1943-1954, 2013.

[8] W. Kawamura, Y. Chiba, M. Hagiwara, and H. Akagi, "Experimental verification of TSBC-based electrical drives when the motor frequency is passing through, or equal to, the supply frequency," 2015 IEEE Energy Conversion Congress and Exposition (ECCE). pp. 5490-5497, 2015

[9] M. Diaz, S. Jose, C. Rica, A. Mora, and F. Rojas, “A novel LVRT control strategy for Modular Multilevel Matrix Converter based highpower Wind Energy Conversion Systems," Ecological Vehicles and Renewable Energies (EVER), 2015 Tenth International Conference on. pp. 1-11, 2015. 